\title{
Two-Dimensional Model of Mechanical Properties of Polymer Blends: Application to Polypropylene and Polyethylene Blends
}

\author{
T. Pakula, M. Kryszewski, J. Grębowicz, and A. Gatęeski \\ Centre of Molecular and Macromolecular Studies, \\ Polish Academy of Sciences, Eódź, Poland.
}

(Received July 10, 1973)

\begin{abstract}
Two new mechanical models for the description of mechanical properties of two-component polymer blends are proposed. On the basis of these models the dependence of the dynamic complex moduli on the blend content is theoretically calculated. Results obtained theoretically are compared with experimental data for polyethylene and polypropylene blends.
\end{abstract}

KEY WORDS Polymer Blends / Rheological Models / Dynamic

Complex Modulus / Diffusion / Miscibility / Phase Inversion /

Both the phase structure of polymer blends and their properties have aroused the interest of a number of investigators. Particular attention has been paid to the mechanical properties of polyblends, in view of their technological significance. Polymer blends, usually two-component and heterogenous systems, cannot, be described in a simple way by the viscoelastic theory of homogenous materials. This in the description of viscoelastic properties of blends a model approach is employed. ${ }^{1-3}$ The mechanical models that are usually used, which differ among themselves with respect to the mode of stress transmission, are one-dimensional and as such do not cover all the aspects of the interactions among the components of the blends. In the study reported here an attempt has been made to describe the viscoelastic properties of polyblends by means of a two-dimensional model. The mathematical description of the models introduced here is the same as the one demonstrated by Sobotka ${ }^{4}$ for diffferent types of mechanical two-dimensional models.

Theoretical results have been used to interpret the composition dependence of the experimental properties of viscoelastic polypropylene (PP) and polyethylene (PE) blends. PP and PE blends were the object of our earlier studies, in which the partial miscibility of the two polymers, conditioned by diffusion processes taking place on the border between the two phases, was demon- strated. $^{5-7}$ Another aim of our study was to establish the effect of diffusion of components on the mechanical properties of the blend.

\section{THEORETICAL CONSIDERATIONS}

The simplest representation of a two-component system is provided by the mechanical models shown in Figure 1, which represent two different mechanisms of stress transmission between the components A and B. The left side of Figure 1 shows a series connection between the components and the right side represents a parallel connection. However, both these models are much too simplified to be suitable for representing a system in which particles of one of the components are dispersed in the other as the medium (Figure 2a). Considering the fact that in the case when such a two-component sample

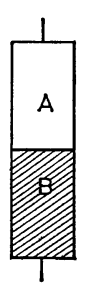

(a)

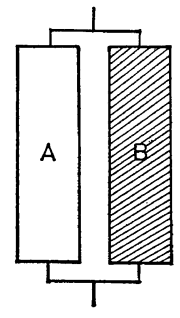

(b)
Figure 1. Mechanical model of series (a) and parallel (b) connection of components. 
(a)

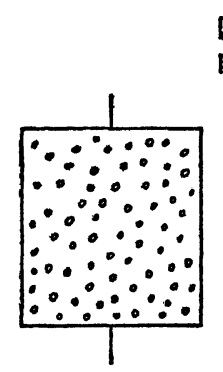

\section{$\square A$}

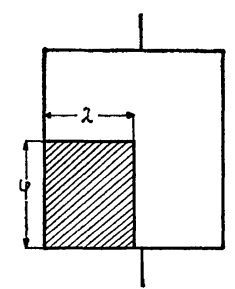

(b)
Figure 2. Models of two-phase structure of blends: (a), Structural model; (b), Mechanical model.

is subjected to tension, some of the lines of force pass only through the component $A$ and some pass through both the components $\mathrm{A}$ and B, then it can be represent with some simplification, by the system presented in Figure 2b. Here, a full cohesion of the components $\mathbf{A}$ and $\mathbf{B}$ is assumed. The values $\lambda$ and $\varphi$ are regarded as parameters representing the mixing state and composition of the sample.

Two simple mechanical models were used in the calculations of the complex modulus of the system shown in Figure $2 b$. These models are presented in Figure 3.

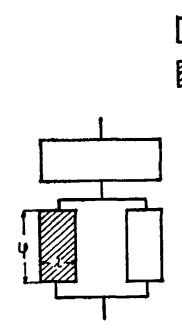

(a)

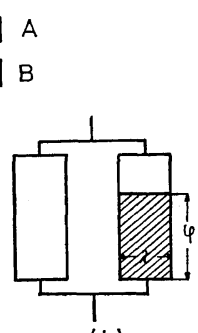

(b)
Figure 3. One-dimensional complex mechanical models of two-component system.

Complex moduli of the strain $E_{\mathrm{a}}{ }^{*}$ for the model (a) and $E_{\mathrm{b}}{ }^{*}$ for the model (b) were calculated in the following way ${ }^{1,2}$ :

$$
\begin{gathered}
E_{\mathrm{a}}{ }^{*}=\left[\frac{\varphi}{(1-\lambda) E_{\mathrm{A}}{ }^{*}+\lambda E_{\mathrm{B}}{ }^{*}}+\frac{1-\varphi}{E_{\mathrm{B}}{ }^{*}}\right]^{-1} \\
{E_{\mathrm{b}}}^{*}=\lambda\left[\frac{\varphi}{E_{\mathrm{B}}{ }^{*}}+\frac{1-\varphi}{E_{\mathrm{A}}{ }^{*}}\right]^{-1}+(1-\lambda) E_{\mathrm{A}}{ }^{*}
\end{gathered}
$$

where $E_{\mathrm{A}}{ }^{*}$ and $E_{\mathrm{B}}{ }^{*}$ are complex dynamic moduli of the components $\mathrm{A}$ and $\mathrm{B}$ respectively. The value $\lambda$ is a measure of the parallel connection of the component $B$ with the whole system, and $\varphi$ is a measure of the series connection of the component B. The product $\lambda \varphi$ is equal to the volume content of the component $B$ in the blend. However, the condition of full cohesion is not satisfied in this model, nor are allowances made for the interactions between the components in the direction perpendicular to the stress applied. Such conditions can be satisfied in a two-dimensional model corresponding to the system in Figure $2 b$. If such a two-dimensional model is to represent the properties of blends it must be an isotropic system in the directions $x$ and $y$ defined by the edges of the model element. This requires an assumption of equality of the values $\lambda$ and $\varphi$, which are denoted by $\alpha$, in which case the content of the component B in the blend has the value $\alpha^{2}$.

In the case of a two-dimensional dynamic deformation of an isotropic viscoelastic body the strain components may be expressed in the following way ${ }^{8}$ :

$$
\begin{aligned}
& \varepsilon_{x}{ }^{*}=C_{1}{ }^{*} \sigma_{x}{ }^{*}+C_{2}{ }^{*} \sigma_{y}{ }^{*} \\
& \varepsilon_{y}{ }^{*}=C_{2}{ }^{*} \sigma_{x}{ }^{*}+C_{1}{ }^{*} \sigma_{y}{ }^{*}
\end{aligned}
$$

where $\varepsilon_{x}{ }^{*}$ and $\varepsilon_{y}{ }^{*}$ are complex values of the relative strains, $\sigma_{x}{ }^{*}$ and $\sigma_{y}{ }^{*}$ are complex values of the stresses.

In the case of a sinusoidally variable strain of a viscoelastic body, the complex values $\varepsilon^{*}$ and $\sigma^{*}$ may be given as:

and

$$
\varepsilon^{*}=\varepsilon_{0} \exp (i \omega t)
$$

$$
\sigma^{*}=\sigma_{0} \exp (i \omega t+i \delta)
$$

where $\omega$ is the angular frequency of deformation, and $\delta$ is the strain-stress-phase-shift angle.

The values $C_{1}{ }^{*}$ and $C_{2}{ }^{*}$ are complex compliance constants which for an isotropic body are related to the tensile complex modulus in along the $x$ - or $y$-direction following manner:

$$
C_{1}{ }^{*}=\frac{1}{E^{*}} \quad \text { and } \quad C_{2}{ }^{*}=-\frac{\nu}{E^{*}}
$$

$\nu$ being a real number corresponding to the Poisson's number for an elastic body.

For simplicity's sake we shall from now on leave out the asterisks denoting complex values, 
though the numbers will still be regarded as such. The properties of the components A and B of the two-dimensional mechanical model presented in Figure $2 \mathrm{~b}$ are represented by complex moduli $E^{\mathrm{A}}$ and $E^{\mathrm{B}}$ or by complex compliances constants $C_{1}{ }^{\mathrm{A}}, C_{2}{ }^{\mathrm{A}}$ and $C_{1}{ }^{\mathrm{B}}, C_{2}{ }^{\mathrm{B}}$ respectively. The assumption of full cohesion between components $A$ and $B$ requires equality of strains in the neighbouring parts of the model. The total strain $\varepsilon_{x}$ of the model element consists of the strain $\varepsilon_{x 1}$ of the component $\mathrm{B}$ and the strain $\varepsilon_{x 2}$ of the component A (Figure 4) in accordance with the equation:

$$
\varepsilon_{x}=\alpha \varepsilon_{x 1}+(1-\alpha) \varepsilon_{x 2}
$$

And in the same way the strain perpendicular to it

$$
\varepsilon_{y}=\alpha \varepsilon_{y 1}+(1-\alpha) \varepsilon_{y 2}
$$

As it is shown in Figure 4 the total stresses $\sigma_{x}$ and $\sigma_{y}$ acting on the model element are given in components as:

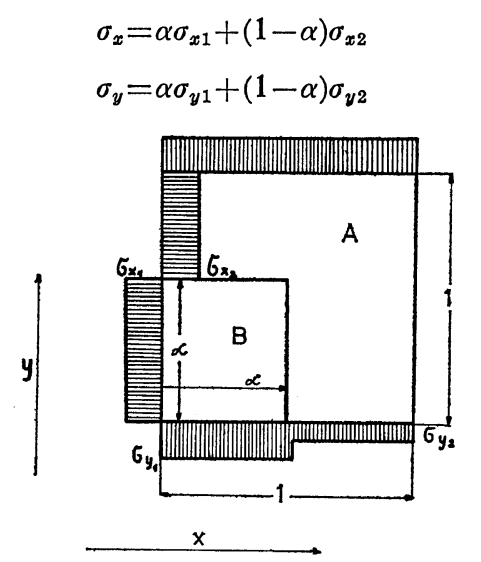

Figure 4. Schematic representation of stress distribution in a simple two-dimensional model.

In our case it is assumed that the model element is subjected to an uniaxial deformation which entails the condition $\sigma_{x}=0$. The eq 9 then, assumes the form:

$$
\alpha \sigma_{x 1}=-(1-\alpha) \sigma_{x 2}
$$

Internally generated stresses $\sigma_{x 1}$ and $\sigma_{x 2}$ as well as $\sigma_{y 1} \sigma_{y 2}$ are the result of unequal values of the moduli $E^{\mathrm{A}}$ and $E^{\mathrm{B}}$ and are also the results of a condition for full cohesion of the two components. Considering such an internal distribu- tion of stress, the stress-strain equations may be expressed ss $^{4}$ :

$$
\begin{aligned}
& \varepsilon_{x 2}=C_{2}{ }^{\mathrm{A}}\left[(1-\alpha) \sigma_{y}+\alpha \sigma_{y 2}\right] \\
& \varepsilon_{y 2}=C_{1}{ }^{\mathrm{A}} \sigma_{y}+C_{2}{ }^{\mathrm{A}} \alpha \sigma_{x 2}
\end{aligned}
$$

The equality of strain in adjacent elements of the components $\mathrm{A}$ and $\mathrm{B}$ entails the following relations:

$$
\begin{aligned}
& \varepsilon_{x 1}=C_{1}{ }^{\mathrm{A}} \sigma_{x 2}+C_{2}{ }^{\mathrm{A}} \sigma_{y}=C_{1}{ }^{\mathrm{B}} \sigma_{x 1}+C_{2}{ }^{\mathrm{B}} \sigma_{y 1} \\
& \varepsilon_{y 1}=C_{1}{ }^{\mathrm{A}} \sigma_{y 2}=C_{1}{ }^{\mathrm{B}} \sigma_{y 1}+C_{2}{ }^{\mathrm{B}} \sigma_{x 1}
\end{aligned}
$$

From eq 10 and 11 we have:

$$
\sigma_{x 1}=-\frac{1-\alpha}{\alpha} \sigma_{x 2} ; \quad \sigma_{y 1}=\frac{\sigma_{y}-(1-\alpha) \sigma_{y 2}}{\alpha}
$$

Substituting the above equation into eq 14 and 15 we can determine the stress components $\sigma_{x 2}$ and $\sigma_{y 2}$ in the following form:

where

$$
\sigma_{x 2}=\frac{P_{2}}{P_{1}} \sigma_{y} ; \quad \sigma_{y 2}=\frac{P_{3}}{P_{1}} \sigma_{y}
$$

$$
\begin{aligned}
& P_{1}=\left[\alpha C_{1}{ }^{\mathrm{A}}+(1-\alpha) C_{1}{ }^{\mathrm{B}}\right]^{2}-(1-\alpha)^{2}\left(C_{2}{ }^{\mathrm{B}}\right)^{2} \\
& P_{2}=\left[\alpha C_{1}{ }^{\mathrm{A}}+(1-\alpha) C_{1}{ }^{\mathrm{B}}\right]\left({C_{2}}^{\mathrm{B}}-\alpha{C_{2}}^{\mathrm{A}}\right) \\
& -(1-\alpha) C_{1}{ }^{\mathrm{B}} C_{2}{ }^{\mathrm{B}}
\end{aligned}
$$

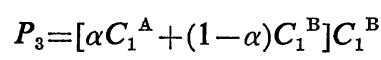

$$
\begin{aligned}
& -(1-\alpha)\left(C_{2}{ }^{\mathrm{B}}-\alpha C_{2}{ }^{\mathrm{A}}\right) C_{2}{ }^{\mathrm{B}}
\end{aligned}
$$

Introducing eq 12-15 into eq 7 and 8 , and taking eq 17 into account, we obtain:

$$
\begin{aligned}
\varepsilon_{y}= & P_{1}{ }^{-1}\left[\alpha C_{1}{ }^{\mathrm{A}} P_{3}+(1-\alpha) C_{1}{ }^{\mathrm{A}} P_{1}\right. \\
& \left.+\alpha(1-\alpha) C_{2}{ }^{\mathrm{A}} P_{2}\right] \sigma_{y} \\
\varepsilon_{x}= & P_{1}^{-1}\left[\alpha C_{1}{ }^{\mathrm{A}} P_{2}+\left(1-\alpha+\alpha^{2}\right){C_{2}}^{\mathrm{A}} P_{1}\right. \\
& \left.+\alpha(1-\alpha) C_{2}{ }^{\mathrm{A}} P_{3}\right] \sigma_{y}
\end{aligned}
$$

Hence it can be said that the complex compliances for a model element are

$$
\begin{aligned}
C_{1}= & P_{1}{ }^{-1}\left[\alpha C_{1}{ }^{\mathrm{A}} P_{3}+(1-\alpha) C_{1}{ }^{\mathrm{A}} P_{1}+\right. \\
& \left.\alpha(1-\alpha) C_{2}{ }^{\mathrm{A}} P_{2}\right] \\
C_{2}= & P_{1}{ }^{-1}\left[\alpha{C_{1}}^{\mathrm{A}} P_{2}+\left(1-\alpha+\alpha^{2}\right) C_{2}{ }^{\mathrm{A}} P_{1}\right. \\
& \left.+(1-\alpha) C_{2}{ }^{\mathrm{A}} P_{3}\right]
\end{aligned}
$$

where $P_{1}, P_{2}$, and $P_{3}$ are given by eq 18 .

The value of the complex dynamic modulus for such a model is given by the expression

$$
E^{*}=\frac{1}{C_{1}}
$$



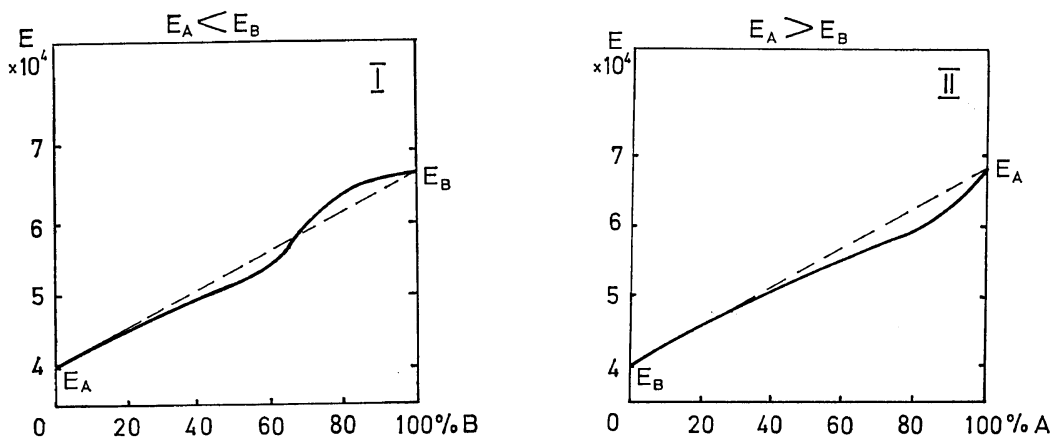

Figure 5. Dependence of modulus on component weight ratio calculated basing on a simple two-dimensional model: (I) $E_{\mathrm{A}}=4 \times 10^{4} \mathrm{Ncm}^{-2}, E_{\mathrm{B}}=6.75 \times 10^{4} \mathrm{Ncm}^{-2}$; (II) $E_{\mathrm{A}}=6.75 \times 10^{4} \mathrm{~N}$ $\mathrm{cm}^{-2}, E_{\mathrm{B}}=4 \times 10^{4} \mathrm{Ncm}^{-2}$. In both cases it is assumed that $\nu_{\mathrm{A}}=\nu_{\mathrm{B}}=1 / 2$.

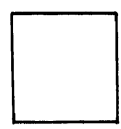

A

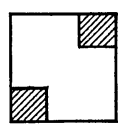

$A: B=9: 1$

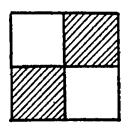

$A: B=5: 5$

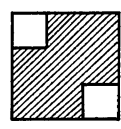

$A: B=1: 9$

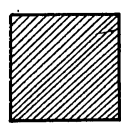

Figure 6. Schematic representation of a complex two-dimensional mechanical model including phase inversion.

The values of $E^{*}$ for definite properties of the components $\mathbf{A}$ and $\mathbf{B}$ have been quantitatively calculated and these results are presented in Figure 5.

The values of the moduli assumed for calculations approximate those obtained experimentally for polyethylene and polypropylene. Curve I in Figure 5 corresponds to a model in which the material of higher modulus value is the phase dispersed in the material of lower modulus value. The curve II describes the inverse case.

The mechanical models of two-component systems presented so far do not reflect the phase inversion phenomon which takes place in blends with comparable contents of both components. This effect can be described by a more complicated model, which is presented in Figure 6, for different content ratios of both components in the blend. The dispersed component is given here by two regions, while the component serving as medium is a continuous element. For equal content of both components their role in the blend is reversed. A mathematical description of the mechanical properties of such a model may be given in the following way.

The whole model element may be divided into

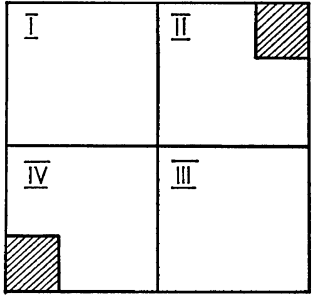

Figure 7. The mode of dividing for a complex two-dimensional model.

four parts, just as in Figure 7, all parts being mutually fully coherent. Parts I and III and parts II and IV have the same mechanical properties, which are, dependent on blend composition. In Figure 7, for example, the properties of parts I and III correspond to the properties of the component A while the properties of parts II and IV may be described by means of the previously presented two-dimensional model depending on the properties of the components A and B. Schematically, the dependence of the moduli of particular parts on blend composition is given in Figure 8. Now, the values $\alpha$ in the two-component parts correspond to 


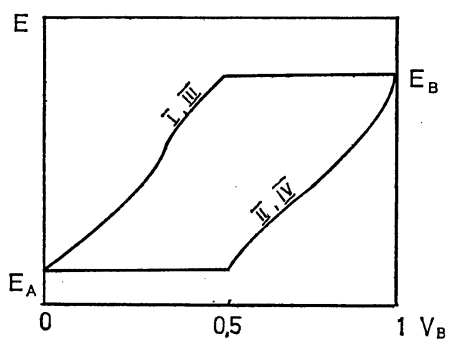

Figure 8. Schematic representation of the changes in moduli of particular parts of the model in Figure 7 vs. composition (weight ratio of components).

$$
\alpha=\sqrt{2 V}
$$

where $V$ is the content of the dispersed component in the blend.

Let $C_{1}{ }^{\mathrm{I}}$ and $C_{2}{ }^{\mathrm{I}}$ stand for the complex compliances of parts I and III of the model and let $C_{1}{ }^{\mathrm{II}}$ and $C_{2}{ }^{\mathrm{II}}$ represent parts II and IV; we can now calculate the compliances of the whole model. Adopting the notations used in our earlier discussion and taking into consideration the symmetry of the model we can formulate the following relations:

$$
\begin{aligned}
\sigma_{y} & =\frac{\sigma_{y 1}+\sigma_{y 2}}{2} \\
\sigma_{x 1} & =-\sigma_{x 2} \\
\varepsilon_{x} & =\varepsilon_{x 1} \\
\varepsilon_{y} & =\varepsilon_{y 1}
\end{aligned}
$$

From the condition of uniform deformation of the adjacent parts we have

$$
\begin{aligned}
& \varepsilon_{x 1}=C_{1}{ }^{\mathrm{I}} \sigma_{x 2}+C_{2}{ }^{\mathrm{I}} \sigma_{y 2}=C_{1}{ }^{\mathrm{II}} \sigma_{x 1}+C_{2}{ }^{\mathrm{II}} \sigma_{y 1} \\
& \varepsilon_{y 1}=C_{1}{ }^{\mathrm{II}} \sigma_{y 1}+C_{2}{ }^{\mathrm{II}} \sigma_{x 2}=C_{1}{ }^{\mathrm{I}} \sigma_{y 2}+C_{2}{ }^{\mathrm{I}} \sigma_{x 2}
\end{aligned}
$$

Formal transformations, similar to the previous ones, yield:

$\varepsilon_{y}=\frac{2\left\{C_{1}{ }^{\mathrm{I}}\left[\left(C_{1}{ }^{\mathrm{II}}\right)^{2}-\left(C_{2}{ }^{\mathrm{II}}\right)^{2}\right]+C_{1}{ }^{\mathrm{II}}\left[\left(C_{1}{ }^{\mathrm{I}}\right)^{2}-\left(C_{2}{ }^{\mathrm{I}}\right)^{2}\right]\right\}}{\left(C_{1}{ }^{\mathrm{I}}+{C_{1}}^{\mathrm{II}}\right)^{2}-\left(C_{2}{ }^{\mathrm{I}}+{C_{2}}^{\mathrm{II}}\right)^{2}} \sigma_{y}$

Hence the value of the complex modulus for this model may be given as:

$$
E^{*}=\frac{\left(C_{1}{ }^{\mathrm{I}}+C_{1}{ }^{\mathrm{II}}\right)^{2}-\left(C_{2}{ }^{\mathrm{I}}+C_{2}{ }^{\mathrm{II}}\right)^{2}}{2\left\{C_{1}^{\mathrm{I}}\left[\left(C_{1}^{\mathrm{II}}\right)^{2}-\left(C_{2}{ }^{\mathrm{II}}\right)^{2}\right]+C_{1}{ }^{\mathrm{II}}\left[\left(C_{1}{ }^{\mathrm{I}}\right)^{2}-\left(C_{2}{ }^{\mathrm{I}}\right)^{2}\right]\right\}}
$$

Figure 9 presents quantitative calculations performed using eq 31 with the values $E^{\mathrm{A}}=4 \cdot 10^{4}$ $N \mathrm{~cm}^{-2}$ and $E^{\mathrm{B}}=6.75 \cdot 10^{4} \mathrm{Ncm}^{-2}$ and $\nu_{\mathrm{A}}=\nu_{\mathrm{B}}=$ $1 / 2$ for different volume ratios of the blend.

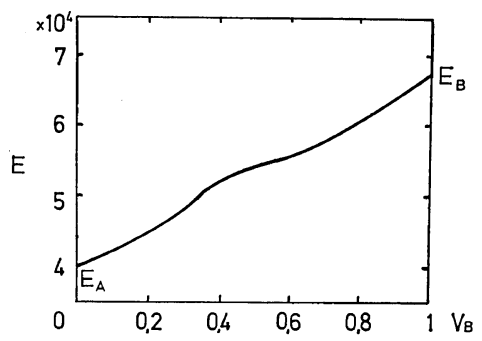

Figure 9. Dependence of modulus vs. component weight ratio for a complex two-dimensional model.

Using eq 31 the stress-strain phase-shift angle for the model presented may be determined. This can be done after the real and imaginary parts of the complex dynamic modulus have been calculated. Then

$$
\operatorname{tg} \delta=\frac{I_{\mathrm{m}}\left(E^{*}\right)}{R_{\mathrm{e}}\left(E^{*}\right)}
$$

\section{EXPERIMENTAL}

\section{Samples}

All experiments were performed on blends of linear polyethylene Lupolen $6000 \mathrm{~L}$ manufactured by BASF and isotactic polypropylene Moplen manufactured by Montecatini. Detailed descriptions of both polymers are available in our previous paper ${ }^{6}$, where a possibility of interdiffusion of macromolecules of these two polymers in their blend was observed. Blends of both polymers of different weight ratios were made as follows: a granulate of both components was mixed in the appropriate weight ratio; the granulate mixture was then pressed at $190^{\circ} \mathrm{C}$, fastcooled in water at room temperature, and granulated for a second time. This procedure was repeated several times. On completion of each cycle of melting and granulation thin cuttings of the blend were examined in an optical microscope in order to determine the degree of mixing of the components. Micrographs for a mixture consisting of 40\% PP and 60\% PE after 


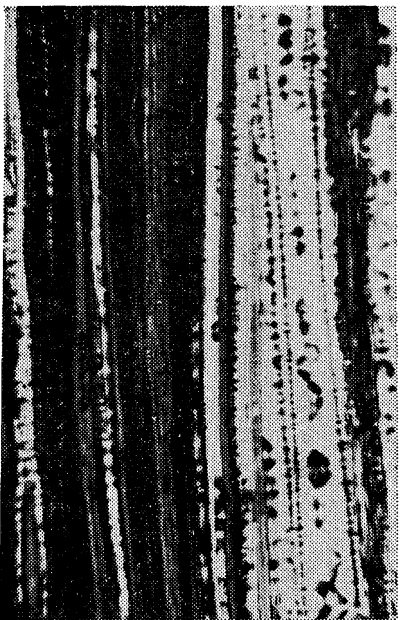

a

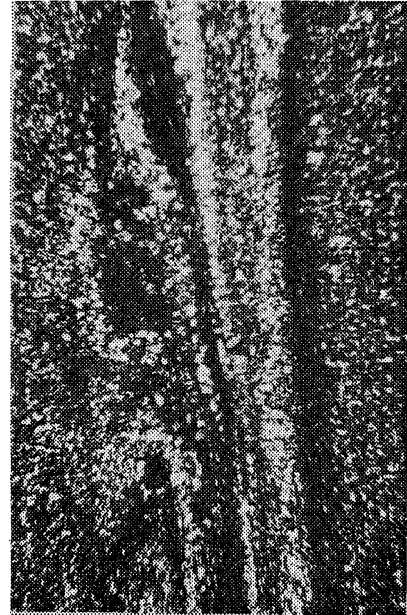

b

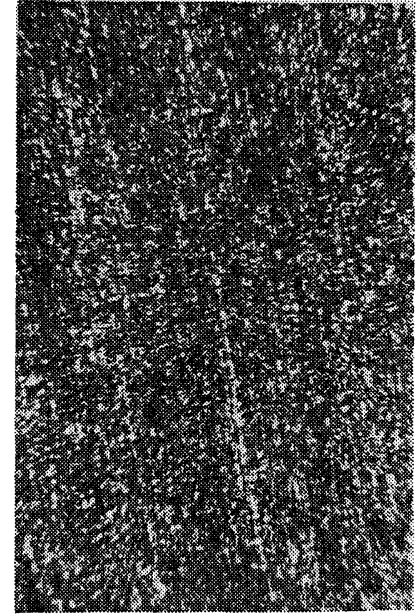

C

$100 \mu$

Figure 10. The change in phase structure of PE and PP blends in consecutive stages of mixing: (a), after three; (b), six; (c), ten cycles of pressing and cooling.

3,6 , and 10 cycles are presented in Figure 10. Samples for which the above procedure was repeated 10 times were found to possess mixing unhomogeneities not exceeding $20 \mu$. Blends obtained in this way were in the form of films $0.5 \mathrm{~mm}$ thick. They were then heattreated in melt at a temperature of $190^{\circ} \mathrm{C}$ under nitrogen for $3.5 \mathrm{hr}$ the aim being a molecular mixing of the two components by way of diffusion. Using a special punch, samples for the investigation of mechanical properties were cut out from both heattreated and unheated films. Both kinds of samples were then cut again, using a microtome, to provide cuttings $10 \mu$ thick for optical microscopy examination. In order to obtain a better differentiation of the supermolecular structures of PE and PP which would make it possible to distinguish between the components of the blend examined in a microscope more precisely, we employed the method (described in our previous study $^{6}$ of thermal treatment of samples. Micrographs showing the structure of both the unheated as well as the heat-treated samples are presented in Figure 11. It appears from Figure 11a that the process of pressing and granulating, repeated ten times, produces a microheterogenous blend over the whole range of weight ratios of the polymers used. This is the state of forced miscibility of the system which is not, however, the state of thermodynamic equilibrium, as is evidenced by changes in the phase structure of the blends in the process of heat-treatment of the melt. Heat-treatment of molten blends gives rise to formation of macroheterogenous systems (Figure 11b) by way of the merging of small, at first dispersed, phase inclusions (the possibility of such a behaviour for systems of limited miscibility has been suggested by several authors). ${ }^{9}$ The interdiffusion of macromolecules of the components used, which occurs simultaneously, is responsible for the fact that each phase of the polyblen is made up of two components. The presence of a dispersed, molecularly diffused component affects the supermolecular structure of the border area of a particular phase; this can be seen in Figure 12, where the structure of PE crystallizing in an unheated blend (Figure 12a) and in a heat-treated one (Figure 12b) are presented for comparison.

\section{RESULTS AND DISCUSSION}

Mechanical measurements were performed using an apparatus of our own design ${ }^{10}$ permitting a sinusoidally variable strain of frequencies ranging from $10^{-3}$ to $10 \mathrm{~Hz}$ to be applied to the sample. The measurements were carried out at $25^{\circ} \mathrm{C}$. Changes in stress and strain in the sample were recorded on an 


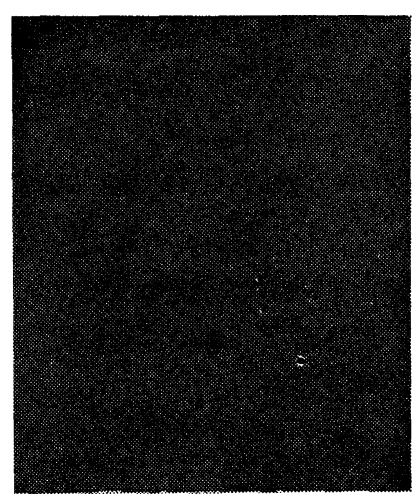

PP

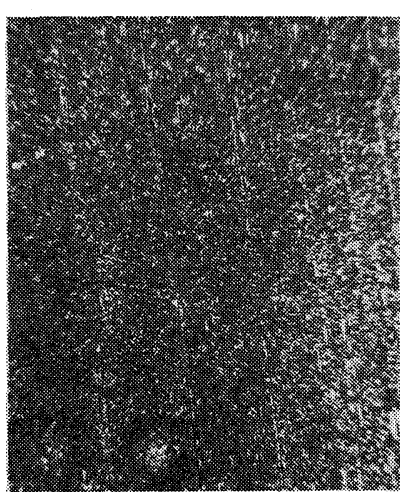

$20 \% \mathrm{PE}$

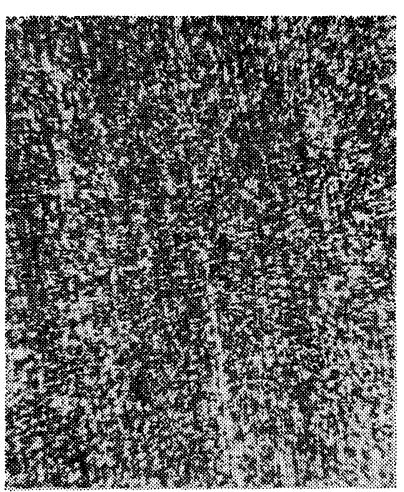

$50 \% \mathrm{PE}$

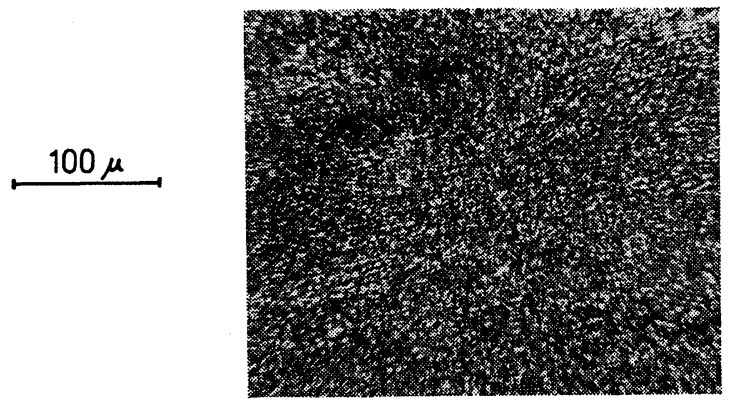

$70 \% \mathrm{PE}$

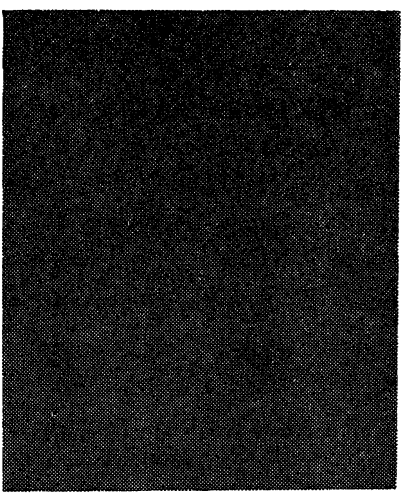

PP

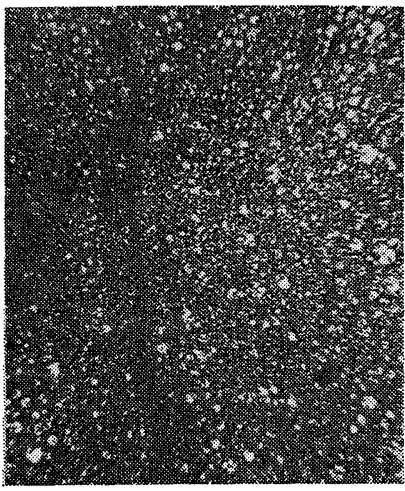

$20 \% \mathrm{PE}$

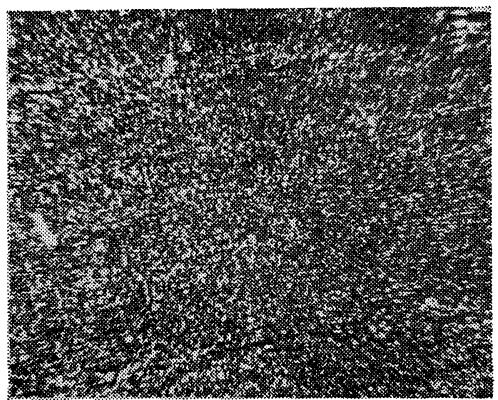

PE

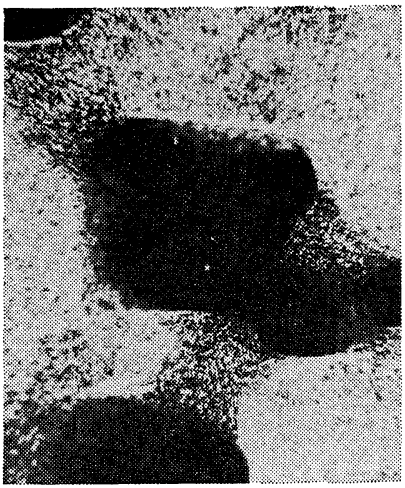

$50 \% \mathrm{PE}$

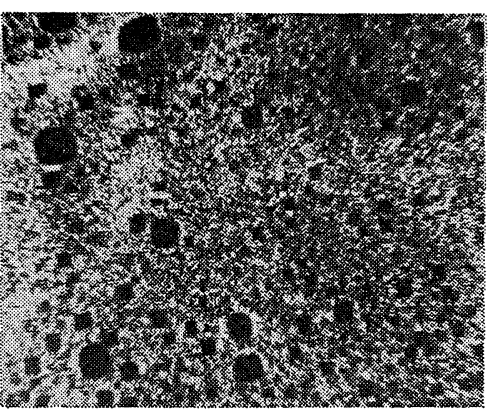

$70 \% \mathrm{PE}$

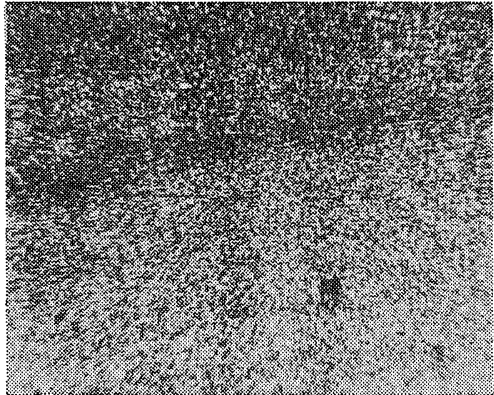

PE

Figure 11. Micrographs of the phase structure in the function of weight ratio of PP and PE blends: (a), unheated samples; (b), heat-treated samples. 
Two-Dimensional Model of Mechanical Properties of Polymer Blends

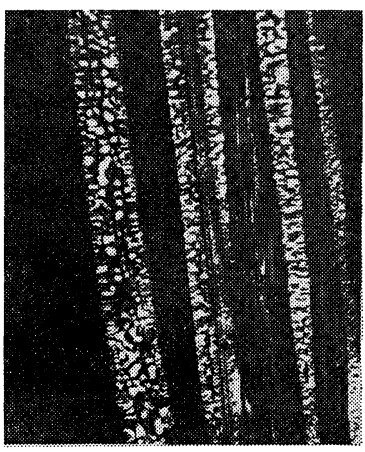

a

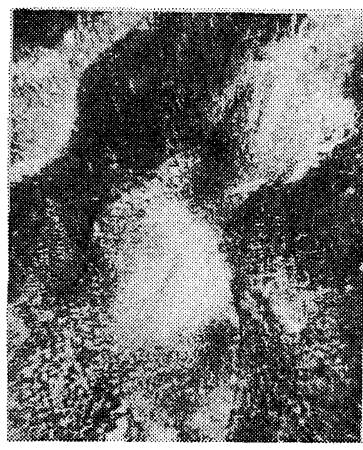

b

$\stackrel{100 \mu}{\longrightarrow}$

Figure 12. Structural changes on the boundary between components after heattreatment of sample.

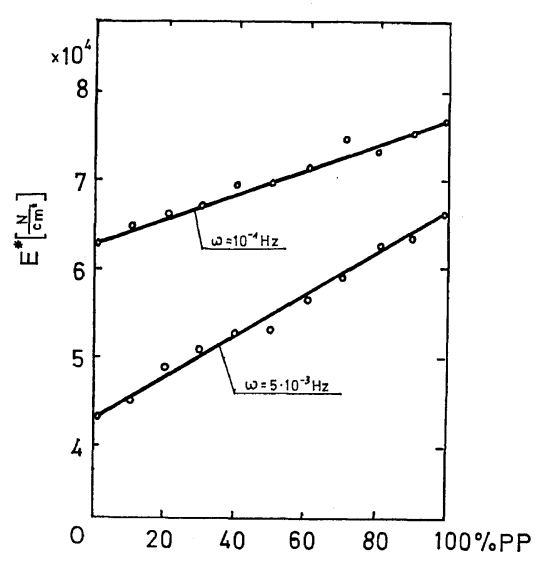

Figure 13. Dependence of modulus on component weight ratio for unheated $\mathrm{PP}$ and $\mathrm{PE}$ blends.

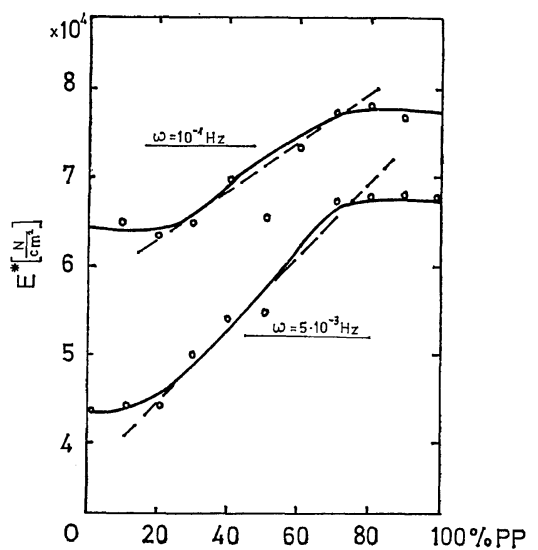

Figure 14. Dependence of modulus on component weight ratio for heat-treated PE and PP blends.

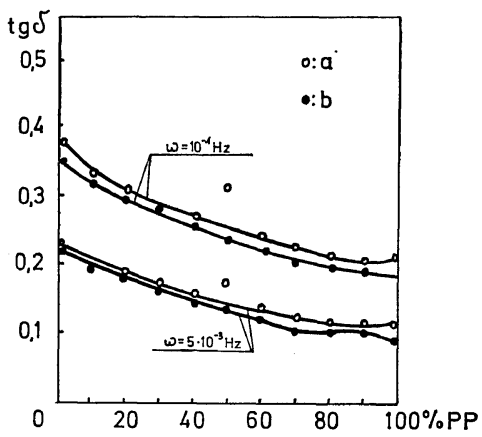

Figure 15. The dependence of $\operatorname{tg} \delta$ on component weight ratio of PE and PP blends: $\bigcirc$, heat-treated samples; O, unheated samples.

oscilloscope or an $X Y$ recorder (depending on frequency) in two mutually perpendicular directions.

Taking advantage of the Lissajous curves obtained, the values of $\left|E^{*}\right|$ and $\operatorname{tg} \delta$ were determined by geometric measurement. The samples were initially stretched up to $10 \%$ of relative strain and variable strain with an amplitude of $2 \%$ of the initial length was applied periodically.* The dependence of the value $\left|E^{*}\right|$ on thecomposition of the blend for unheated samples for frequencies of $5 \times 10^{-3} \mathrm{~Hz}$ and $10^{-1} \mathrm{~Hz}$ is plotted in Figure 13. Both these plots hardly deviate from a straight line between the values of $\left|E^{*}\right|$

* The effect of static stress relaxation was observed. However, the measurements were taken at sufficient time intervals after starfing the vibrations that a steady state was reached. 
for PP and PE for given deformation frequencies. Thus, the effect of frequency on the character of the dependence of the dynamic modulus on the blend composition cannot be observed here. The dependence for heat-treated samples was found to be slightly different. Results obtained for the same frequencies are given in Figure 14. In this the deviations from a straight line are greater. Figure 15 presents the dependence of $\operatorname{tg} \delta$ on the blends composition for the two kinds of samples: heat-treated and unheated. It is seen from the results presented that heat-treatment of the molten blends has a marked effect on the dependence of their mechanical properties on blend composition. A comparison of the results shown in Figure 13 for unheated samples with Figure 9, which shows the theoretical dependence of dynamic the modulus for a two-dimensional model in which phase inversion has been taken into consideration, leads one to the conclusion that basically, the two dependences do not differ very much. Both experimental results as well as theoretical dependences deviate from a straight line only slightly. It follows that the mechanical properties of a well-dispersed heterogeneous two-component blend may be described adequately by means of eq 31 .

The results of theoretical calculations for this model do not, however, describe directly the dependences observed for heat-treated samples. Adopting, however, the model postulated in our previous study ${ }^{6}$ of phase structure of blends in which limited diffusion of both components is taken into account it can be supposed that heattreated blends are two-phase systems in a limited range of component weight ratios. In such a case a mechanical model of a two-component blend may also be used only in a limited range of weight ratios. It follows from Figure 14 that the dependence of the modulus $\left|E^{*}\right|$ on composition may be approximated for a volume ratio from about 20 to $70 \%$ of PE content in the blend by means of a straight line. This suggests that in such a range of weight ratios the blends studied are two-component systems and their mechanical properties may be described by means of the mechanical model suggested. The agreement obtained is an interesting result but it must be confirmed by a greater number of experiments. Interpretation of results in the way presented here may provide a useful criterion for the estimation of the miscibility of polyblend components.

\section{REFERENCES}

1. M. Takayanagi, H. Harima, and Y. Iwata, $J$. Soc. Mat. Sci. (Japan), 12, 389 (1963).

2. K. Fujino, Y. Ogawa, and H. Kawai, J. Appl. Polym. Sci., 3, 2147 (1964).

3. T. Okamoto and M. Takayanagi, J. Polym. Sci., Part C 23, 597 (1968).

4. Z. Sobotka, J. Makromol. Sci., B 5, 393 (1971).

5. M. Kryszewski, T. Pakuła, and J. Grębowicz, Vysokomol. Soedin., in press.

6. M. Kryszewski, A. Gałęski, T. Pakuła, and J. Grębowicz, J. Colloid Interfac. Sci., in press.

7. J. Letz, J. Polym. Sci., Part A-2, 7, 1987 (1969); ibid., Part A-2, 8, 1415 (1970).

8. Turner Alfrey J., and E. F. Gurnee in "Rheology-Theory and Applications," E. R. Eirich, Ed., Pergamon Press, New York, 1956 Vol. 1, Chapter 11.

9. W. Je. Gul, Vysokomol. Soedin., 14, 291 (1972).

10. T. Pakuła, Z. Soukup, and M. Kryszewski, $J$. Polym. Sci., (Polymer Phys.), in press. 